\title{
OSTEOARTHRITIS
}

\section{Activate autophagy to prevent cartilage degeneration?}

Pharmacological activation of autophagy might prevent age-related degenerative changes in cartilage that are associated with osteoarthritis (OA), according to researchers from La Coruña, Spain. Although defective autophagy and mitochondrial dysfunction were known to coexist in mouse models of age-related and surgically induced $\mathrm{OA}$, new findings show that mitochondrial function and autophagy regulation in chondrocytes are directly linked-via the Akt-mTOR (mammalian target of rapamycin) signalling pathway.

"Our most important finding was that autophagy plays a critical role in protecting chondrocytes from oxidative stress," notes Beatriz Caramés, the study's corresponding author. The researchers observed that autophagy decreases in response to mitochondrial dysfunction (induced by oligomycin, an inhibitor of the electron transport chain) in normal chondrocytes. $24 \mathrm{~h}$ after oligomycin treatment, expression of autophagy markers increased, then decreased after $48 \mathrm{~h}$, eventually leading to cell death. However, inducing autophagy using mTOR inhibitors, prior to oligomycin treatment, protected chondrocytes against both mitochondrial damage and apoptosis. Caramés and colleagues suggest that the transient increase in autophagy represents a compensatory response to cell stress, and that damage occurs when prolonged stress exceeds the capacity of this mechanism.

Moreover, cells in which autophagosome formation was prevented (by silencing expression of autophagy protein 5) showed increased production of reactive oxygen species, further highlighting the interdependence of autophagy and mitochondrial function.

The researchers hope their work will lead to new treatments for age-related musculoskeletal disorders, including OA. "We have found promising targets," says Caramés. "We now aim to impact the OA

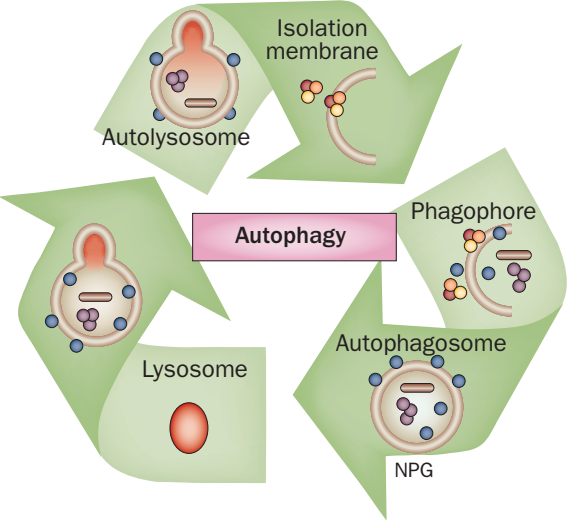

therapeutic landscape by validating those targets using relevant small molecules and biologics in therapeutic contexts."

Caroline Barranco

Original article López de Figueroa, P. et al. Autophagy
activation protects from mitochondrial dysfunction in
human chondrocytes. Arthritis Rheumatol. doi:10.1002/
art.39025

